\title{
Securitization of Disinformation in NATO Lexicon: A Computational Text Analysis
}

\author{
H. Akın Ünver \\ Department of International Relations \\ Özyeğin University \\ İstanbul, Turkey \\ akin.unver@ozyegin.edu.tr
}

\section{A Preprint}

\author{
D Ahmet Kurnaz \\ Department of Public Administration and Political Science \\ Çanakkale Onsekiz Mart University \\ Çanakkale, Turkey \\ ahmetkurnaz@hotmail.com
}

February 21, 2022

\begin{abstract}
Abstract: Following the Russian meddling in the 2016 US elections, disinformation and fake news became popular terms to help generate domestic awareness against foreign information operations globally. Today, a large number of politicians, diplomats and civil society leaders identify disinformation and fake news as a primary problem in both domestic and foreign policy contexts. But how do security institutions define disinformation and fake news in foreign and security policy, and how do their securitization strategies change over years? This article explores 238,452 tweets from official NATO and affiliated accounts, and more than 2,000 NATO texts, news, statements, and publications using computational methods since January 2014 and presents an unsupervised structural topic model (stm) analysis to explore the main thematic and discursive contexts of these texts. The study finds that NATO's threat discourse and securitization strategies are heavily influenced by US political lexicon and discovers that word choices change based on their likelihood of mobilizing alliance resources and cohesion. In addition, the study finds that NATO's recent disinformation agenda is in fact a continuity of NATO's long-standing Russia-focused securitization discourse and an attempt to mobilize alliance attention on Baltic states and Poland to counter Russia.
\end{abstract}

Keywords disinformation $\cdot$ NATO $\cdot$ Securitization $\cdot$ structural topic model 


\section{Introduction}

Over the last few years, disinformation, information warfare and fake news have become important strategic

and political concepts in international relations (Cour la, 2020; Lanoszka, 2019). Although these terms aren't new and are as old as the term 'propaganda', their mainstream use within the context of digital communication skyrocketed after the 2016 US elections (Grinberg et al., 2019). However even before that, these terms had begun to enter into the foreign policy discourse of NATO countries following the 2014 Russian military operations in Ukraine. Prior to the annexation of Crimea, Russia had already designated information warfare as part of its 2010 Military Doctrine, which was updated again 2014 with a special emphasis on digital communication (Renz, 2014). A year prior, the importance of the digital space for military doctrinal considerations was outlined by General Valery Gerasimov - Russian Chief of the General Staff. In his 2013 article titled 'The Value of Science is in the Foresight', Gerasimov wrote: "The very 'rules of war' have changed. The role of nonmilitary means of achieving political and strategic goals has grown, and, in many cases, they have exceeded the power of force of weapons in their effectiveness. ... All this is supplemented by military means of a concealed character." (Galeotti, 2019) These three texts are generally accepted as the doctrinal basis of modern Russian information operations that were demonstrated both in Ukraine in 2014, and later in Syria, after mid-2015 (Sinovets, Renz, 2015).

Since then, strategic communicative actions that are intended to influence, mislead and confuse foreign populations have assumed a central position in global debates about politics and foreign policy. Given the impact of such actions on elections, polarization and crisis management, it was natural for the rhetoric about these actions to assume such a central position(Bradshaw, Howard, 2018). However, over time, popular buzzwords like 'disinformation/misinformation', 'fake news', and 'information operations' have proliferated in global political mainstream discourse and assumed an accusatory nature worldwide as more leaders, diplomats, and politicians have begun using them to discredit and delegitimize their political opponents. This dynamic was later conceptualized as 'discursive deflection' (Ross, Rivers, 2018; Smith, 2019) and became acutely visible in the foreign policy domain, as more countries have begun securitizing the concepts 'fake news', 'disinformation', and 'information warfare' to similarly discredit, and delegitimize rival countries Baum, Potter, 2019). Broadly speaking, 'discursive-deflection' is the strategy of discrediting competitors and rivals by portraying oneself as the sole source of truth. While the domestic political use of these terms is well-studied, we are still somewhat in the dark with regard to why countries choose to securitize these terms and what happens in their interactions with other countries when they do so.

The foreign policy use of such terms predates the 2016 US elections and proliferated after the Russian military involvement in Crimea and Donbas (Khaldarova, Pantti, 2016). The primary reason for this contextual proliferation was the Russian decision to deny the initial stages of its involvement in Ukraine and its broader strategy of distracting and dividing Western attention over Russian military operations (Mejias, Vokuev, 2017). There is still a debate over whether it was really Russian information operations that had derailed NATO response in Ukraine, or if disinformation discourses are employed in order to shift the blame over to Russia at a time when NATO was already divided over its commitment to Ukraine (Lysenko, Brooks, 2018). While there is robust evidence of Russian information operations in Ukraine and its role in spreading 
disinformation in NATO countries, sustained NATO apathy towards rising Russian military influence in the Black Sea after 2014 and Syria after 2015 support the latter claim.

Critics of Western disinformation discourses for example, argue that such discourses have turned into 'floating (or empty) signifiers', that have no specific or agreed-upon meaning (Farkas, Schou, 2018). In that vein, blaming others for engaging in disinformation often distracts attention away from a mistake or failed policy enacted by the blamer (Monsees, 2020). In this case, critics argue that Western discourses on disinformation are intended to distract attention away from NATO or EU divisions, or more domesticlevel polarization dynamics, by creating a unique empty signifier (disinformation) that is employed as a rallying rhetoric that bolsters the significance of external threats (Mas-Manchón et al., 2021). This way, disinformation and its associated terms like misinformation, fake news and information war get securitized, receiving disproportionate levels of attention in the policy domain. In this context, disinformation and its associated terms are used to exaggerate an existing threat and create a rallying discourse that would channel the attention of the divided Western nations away from their internal disagreements, and towards an inflated external threat. Some scholars go even further, arguing that disinformation is being securitized in the West (especially in NATO) to the extent the 'war on terror' was securitized through the 2000s (Lanoszka, 2019). In this line, disinformation is alleged to have become a new strategic glue that would help Western nations pool in their increasingly diverging interests and resources into a common cause (Baumann, 2020).

Securitization of disinformation in domestic politics is relatively well-studied (Freelon, Wells, 2020; Neo, 2021). Although these terms have entered into mainstream debate after the 2016 US elections, former President Donald Trump too, had securitized fake news to delegitimize his opponents by constructing rival disinformation as a national security problem, indirectly attributable to China (Polletta, Callahan, 2019). Following the tornado of accusations in the US, political actors in Britain, France, Italy, South Africa, Kenya and others have begun blaming each other for engaging in organized disinformation (Maweu, 2019; Saurwein, Spencer-Smith, 2020). Even in Sweden, there is empirical evidence that suggests accusing journalists of spreading fake news results in self-censorship of such outlets (Bennett, Livingston, 2018). There are further cases of evidence supporting the claim that elite-level discourses on disinformation have a direct effect on how the wider society perceives information and facts in general, creating a measurable effect on public trust towards such facts and information (Van Duyn, Collier, 2019). In Singapore for example, delegitimizing rival parties and news outlets through disinformation discourse is considered 'acceptable', as part of the state's duty to discipline the opposition and its political actors (Tan, 2020). Similar trends emerging in democracies and authoritarian countries alike, such as in Austria, Australia, Poland, Russia, and South Africa, demonstrate the universality of instrumentalizing disinformation discourse as a political delegitimization tactic (Kurowska, Reshetnikov, 2018; Tandoc et al., 2018).

While a robust scholarship is emerging on the domestic political uses of disinformation discourse, there has so far been no longitudinal, large-N study that explored how such constructions emerge in international politics. Furthermore, there has so far been no exploration of how such discourses evolve over time and under what contexts in foreign affairs. We know that disinformation and fake news are important issues in world politics and that they are frequently used to bring an issue to public attention, but we are more in the 
dark over the contextual and temporal nuances that drive how these concepts are discursively constructed in foreign policy discourse.

This study aims to provide an early addition to the emerging literature on foreign policy uses of disinformation discourses by focusing on how the North Atlantic Treaty Organization (NATO) has used them in its documents and social media posts. It does so by studying 238,452 tweets from official NATO and affiliated accounts, and more than 2,000 NATO texts, news, statements, and publications using computational methods since January 2014 and presents an unsupervised structural topic model (stm) analysis to explore the main thematic and discursive contexts of these texts. Ultimately, we hope to trigger a wider debate on the securitization of disinformation and fake news in foreign policy, and the greater explanatory value of computational methods in studying large- $\mathrm{N}$ text data in studying such securitization strategies.

\section{Securitizing Disinformation}

Over the last few years, defining what misleading content is and how to measure the legitimacy of its dissemination has been at the forefront of journalistic, political and scientific debate (Tandoc et al., 2018). Even before its proliferation in 2016, disinformation was a widely-used term in the mainstream discourse, co-existing with other terms such as infoglut, or information overload (Andrejevic, 2013). Although at first disinformation and misinformation were terms used interchangeably, today, disinformation refers to the deliberate dissemination of false information with the intention to mislead and confuse its audiences. Misinformation, on the other hand, strictly defines unintended diffusion of false information by mistake, without malintent. There are also bridge terms such as 'malinformation' that is factually accurate but is deployed to harm and damage an individual or an entity, or the concept of 'problematic information' as defined by Caroline Jack (Jack, 2017; Morgan, 2018). Although it is not directly mentioned, all of these concepts refer to the digital space, where information manipulation is disseminated faster and wider on social media and digital communication technologies compared to more media forms of media.

As terms 'disinformation/misinformation', 'fake news', 'information operation', and 'hybrid war' are often used interchangeably in political discourse, there are no clear-cut differences in the strategic topic of each word choice (Guess, Lyons, 2020). Politicians and leaders can often use these terms as a bag of buzzwords, without a clear operational definition of what each of them precisely means. Each of these buzzwords generate roughly the same effect on the consumers of such messages, which is the delegitimization of their target (Tucker et al., 2018). Especially problematic is the fact that once the discourse on disinformation is weaponized to delegitimize rivals, there is very little such rivals can do to defend themselves. Given the significant political charge of these terms, individuals or institutions that are alleged to be engaging in disinformation-related activities often have to enter into a fruitless spar of words to challenge such allegations, which usually leads to further controversy. This renders the accuser - or the side that securitizes disinformation - more advantageous compared to the accused, generating a dynamic similar to the 'attacker's advantage' in cyber security where the defender is continuously blindsided (Tong et al., 2020).

Therefore, the securitization of disinformation - that is, discursively constructing disinformation as a security concern - is becoming almost as controversial as disinformation itself, and can often be deployed 
to muddle the waters of a healthy debate. Its problem lies within its success; namely how successfully disinformation gets securitized and rallies policy resources around itself. This fits into Buzan et. al. criteria for a 'successful speech-act', which takes places in a medium most appropriate for its dissemination and have a clear, mobilizable referent object (i.e. 'those that spread disinformation') (Buzan et al., 1998). By securitizing disinformation in the medium that is most conducive for its dissemination (social media and Internet), speakers get a chance to use the speed and volume advantage of digital communication technologies against their opponents. Also, such discursive constructions must be sedimented (1) rhetorically: have a clear argumentative function, (2) discursively: contain clear power and hegemonic relations within, (3) culturally: refer to a well-known case or instance, and (4) institutionally: in a way that mobilizes policy resources (Williams, 2003).

Yet for the Copenhagen school, not all speech acts constitute securitization. Securitization is a very particular discursive construct that designates a specific existential threat that requires the mobilization of uncommon resources and measures that go beyond the norms of institutional and political responses (Knudsen, 2001). In many cases, securitization happens to trigger and facilitate these institutional changes by 'shocking' power brokers and bureaucracies into action either through internal bureaucratic peer pressure, or through public opinion pressure (audience costs). As such, disinformation has been lifted 'above politics' in Western rhetoric as a peculiar threat that requires a sidelining of daily political squabbles, mobilizing unique resources and addressing it in unity that would otherwise cannot materialize (Buzan, Wæver, 2009). Ultimately the discursive constructions of disinformation do constitute acute cases of securitization as they generate amity-enmity relations among countries that adopt this discursive strategy, versus those that do not (Buzan, Wæver, 2009).

Social media offers a unique challenge for the study of securitization. Traditionally, such securitizing statements are extracted from lengthy speeches and texts through discourse analysis. However, the advent of faster and higher-volume digital communication technologies have led to a shift of state and elite discourses from older to newer media systems (Balzacq et al., 2016). To that end neither Facebook, nor Twitter or Instagram provides contiguous discursive framing opportunities for researchers to study securitization dynamics due to their word limits (Bouvier, Machin, 2018). Furthermore, since elite and state-level discourses on social media are often written by assistants, communications representatives or PR firms, they don't constitute 'performative actions' that are the cornerstone of securitization (Lipizzi et al., 2016). This generates a significant 'context gap', where researchers may not fully understand the wider thematic and lexical ecosystem that such social media posts may inhabit. Interpreting securitization in such media platforms thus necessitates more robust techniques of 'horizon scanning' that allows researchers to extract long-term discursive variances and contexts.

Computational text analysis methods largely deliver this horizon scanning. Where social media posts lack in word and character limits, they provide an immense volume of data that provides ample context in longitudinal analysis. By extracting large quantities of text data from social media, researchers can not only interpret the changing contours and contexts of securitization, but they can also cross-check these findings with more traditional forms of discursive construction outlets such as speeches, documents and archival 


\begin{tabular}{lllll}
\hline Type & All & Disinfo & Disinfo Ratio & Rest \\
\hline tweet & 238452 & 4112 & 1.72 & 234340 \\
speeches & 1136 & 223 & 19.63 & 913 \\
press_releases & 1083 & 12 & 1.11 & 1071 \\
thematic_topics & 142 & 11 & 7.75 & 131 \\
reviews & 119 & 44 & 36.97 & 75 \\
official_texts & 19 & 4 & 21.05 & 15 \\
archives & 16 & 0 & 0.00 & 16 \\
publications & 12 & 9 & 75.00 & 3 \\
basic_texts & 10 & 4 & 40.00 & 6 \\
\hline
\end{tabular}

Table 1: Descriptive statistics of the text dataset. 'All' denotes the aggregate number of contents within that specific document type. 'Disinfo' denotes the number of documents that contain disinformation-related keywords within. 'disinfo_ratio' denotes the proportion of documents that contain disinformation-related keywords within the broader pool of documents analyzed.

material. That is why in this study, we not only engage in a large-scale longitudinal 'old form' securitization analysis by focusing on NATO archives, but also add in 'new form' analysis by extracting a large tweet dataset of official NATO accounts.

The logic of interpreting how disinformation gets securitized through relying on NATO documents is two-fold: first, NATO has been evolving to find new raisons d'être after the end of the Cold War and sought to capitalize on the securitizations of new forms of threats, such as terrorism, cybersecurity, Syria and forced migration (Stritzel, Chang, 2015). Disinformation and information war are two of the recent additions to this threat portfolio and helps us understand how NATO's discourses on security adapt to a new-medium threat. Second, it enables us to understand how institutional security arrangements like NATO reinvent their security identities and construct their amity-enmity relations in light of newer technologies. Since identity and action are considered closely linked in constructivism, and that they are never fixed or intrinsic but rather fluid and are constituted through social processes, studying longitudinal securitization dynamics gives us valuable insight over long-term NATO security planning (Mälksoo, 2018).

\section{Methodology}

Since this study is interested in the longitudinal dynamics of how disinformation and related terms were securitized, and since the volume of text that we are dealing with is quite large, we follow a computational methodology that combines social media text data extraction methods with traditional text analysis tools. In recent years social media data has grown into a useful study area for social scientists as more and more governmental documents become digitized and such governments start taking an active role on social media (DiMaggio, 2015). Although traditional forms of text analysis and discourse analysis approaches use hand coding schemes, newer methods in text mining and analysis are increasingly more preferred due to their ability to process large quantities of text data and eliminate the inter-coder reliability issues from the equation (Krippendorff, 2004). Moreover, these newer methods increase the causal robustness of text data by building inter- and intra-text causal inferences, strengthening the explanatory power of words as dependent or independent variables. 


\begin{tabular}{ll}
\hline Type & $\mathrm{N}$ \\
\hline speeches & 351 \\
reviews & 63 \\
press_releases & 14 \\
basic_texts & 6 \\
thematic_topics & 15 \\
official_texts & 6 \\
publications & 17 \\
tweet & 4302 \\
\hline
\end{tabular}

Table 2: The number of occurrences for disinformation-related keywords in each document type

In order to explore how NATO has securitized disinformation in recent years, we have scraped 238,452 tweets from NATO and official affiliated accounts from January 2014 to February 2021 and extracted more than 2000 speeches, press releases, reviews, official texts, archival documents and publications from the NATO e-library (?). Out of this sample, we have extracted documents and content that contained the keywords 'disinformation', 'misinformation', 'fake news', 'propaganda', 'hybrid warfare' and 'information warfare', and logged the number of their occurrences within these texts by date. Since this study doesn't focus on the semantic differences between these keywords and consider them as different references to disinformation as a discursive strategy, we code and merge them singularly as variable 'disinfo'. Our preliminary analysis shows that NATO has used these keywords most frequently in tweets, followed by speeches, reviews and publications. However, when analyzed proportionally, NATO publications focus on disinformation most frequently ( $75 \%$ of all documents), followed by tweets (40\%), reviews (36.97\%) and official texts (21.05\%).

In this study, we employ structural topic modeling (STM) - a text analysis approach that finds 'topics' in an unstructured corpus based on covariate information (Roberts et al., 2019). It follows a statistical logic that measures co-occurrence likelihoods of keywords and terms that are more likely to appear with each other and derives topical meanings out of those likelihoods. Topic modeling is increasingly used in the study of large volumes of text in social sciences such as large archival documents, or social media text datasets by producing "each word on the basis of some number of preceding words or word classes," and "generate[ing] words based on latent topic variables inferred from word correlations independent of the order in which the words appear" (Wallach, 2006). In recent years topic modeling has become a widely-used method to study large Twitter datasets, and political discussions that happen on other social media platforms (Giles, 2012; Hong, Davison, 2010; Liang, Fu, 2015).

A longitudinal analysis of the specific keywords sorted by document type reveals a clear difference in word choices between different NATO documents. In NATO Basic Texts, the most-preferred reference keyword is 'hybrid warfare', whereas in press releases reliance on the word 'misinformation' gradually evolves into 'disinformation' by 2018. NATO reviews also largely prefer 'misinformation', but NATO speeches and tweets are more diverse with a heavier use of the term 'propaganda', 'disinformation' and 'fake news'. This difference is an interesting demonstration how elastic these terms are and how different institutional cultures and outlets can prefer one over the other in their communication strategies. 


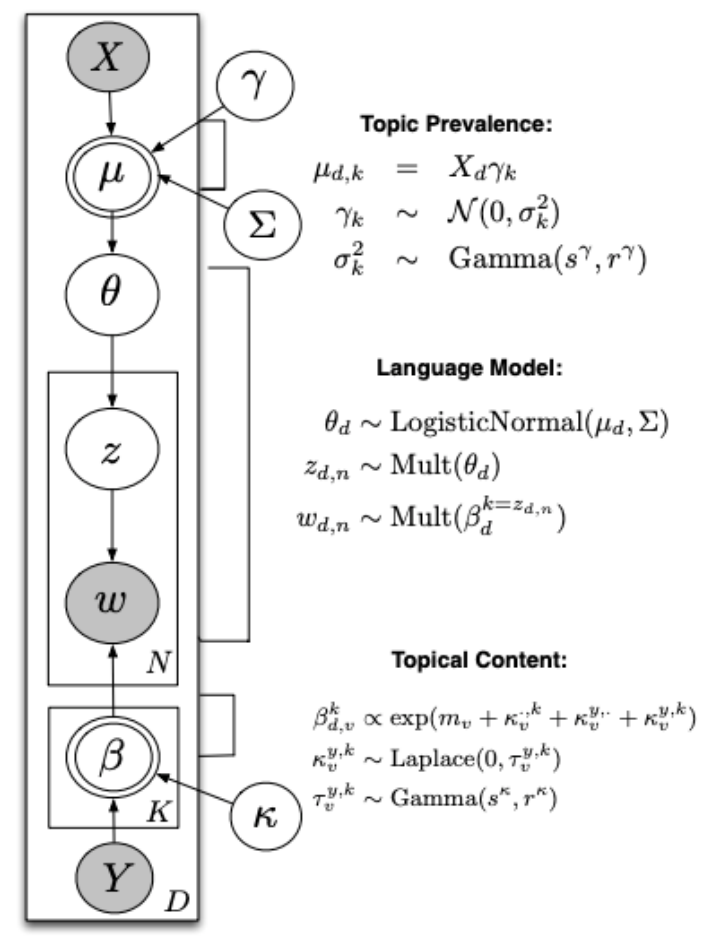

Figure 1: : Plate Diagram for Structural Topic Model: "The model combines and extends three existing models: the correlated topic model (CTM), the Dirichlet-Multinomial Regression (DMR) topic model and the Sparse Additive Generative (SAGE) topic model. The logistic normal prior on topical prevalence in the standard CTM is replaced by a logistic-normal linear model. The design matrix for the covariates X allows for arbitrarily flexible functional forms of the original covariates using radial basis functions (our R package also provides B-splines). The distribution over words is replaced with a multinomial logit such that a token's distribution is the combination of three effects (topic, covariates, topic-covariate interaction) operationalized as sparse deviations from a baseline word frequency $(\mathrm{m})$. Our software provides the analyst with a choice of regularizing priors for the GLM coefficients $(\kappa, \gamma)$ with defaults: Normal-Gamma prior pooled by topic for $\gamma$ and the "Gamma Lasso" prior [10] for $\kappa$ (Roberts et al., 2013)

The difference between NATO official texts and tweets are particularly interesting. Although NATO official texts shift from a 'hybrid war' focused discourse to 'disinformation' focused discourse by 2018, the reliance on 'disinformation' discourse in tweets is more striking. By late-2016 (US elections) 'disinformation' becomes a clear discursive choice in NATO tweets, skyrocketing in much of 2020, due to COVID and vaccinerelated securitization discourses globally. This could be interpreted as the discursive anchoring capacity of the United States for NATO, as the constructions of securitization in American political culture affects the wider institutional discourse of NATO. Perhaps as the clearest sign of the temporal variations in word choice shifts, NATO's Twitter accounts overwhelmingly use 'disinformation', 'information warfare', and 'misinformation words in comparison to its official texts and statements, that rely on 'cyber war' and 'hybrid war'. As for NATO and affiliated accounts that use the keyword 'disinformation', three clear accounts stand out. These are @STRATCOMCOE (NATO Strategic Communications Centre of Excellence), @NATOmoscow (NATO Information Office Moscow), @NATOBrazeB (NATO Assistant Secretary General for Public Diplomacy), and @NATOpress (Official Twitter account of the @NATO Spokesperson). As for which NATO country 


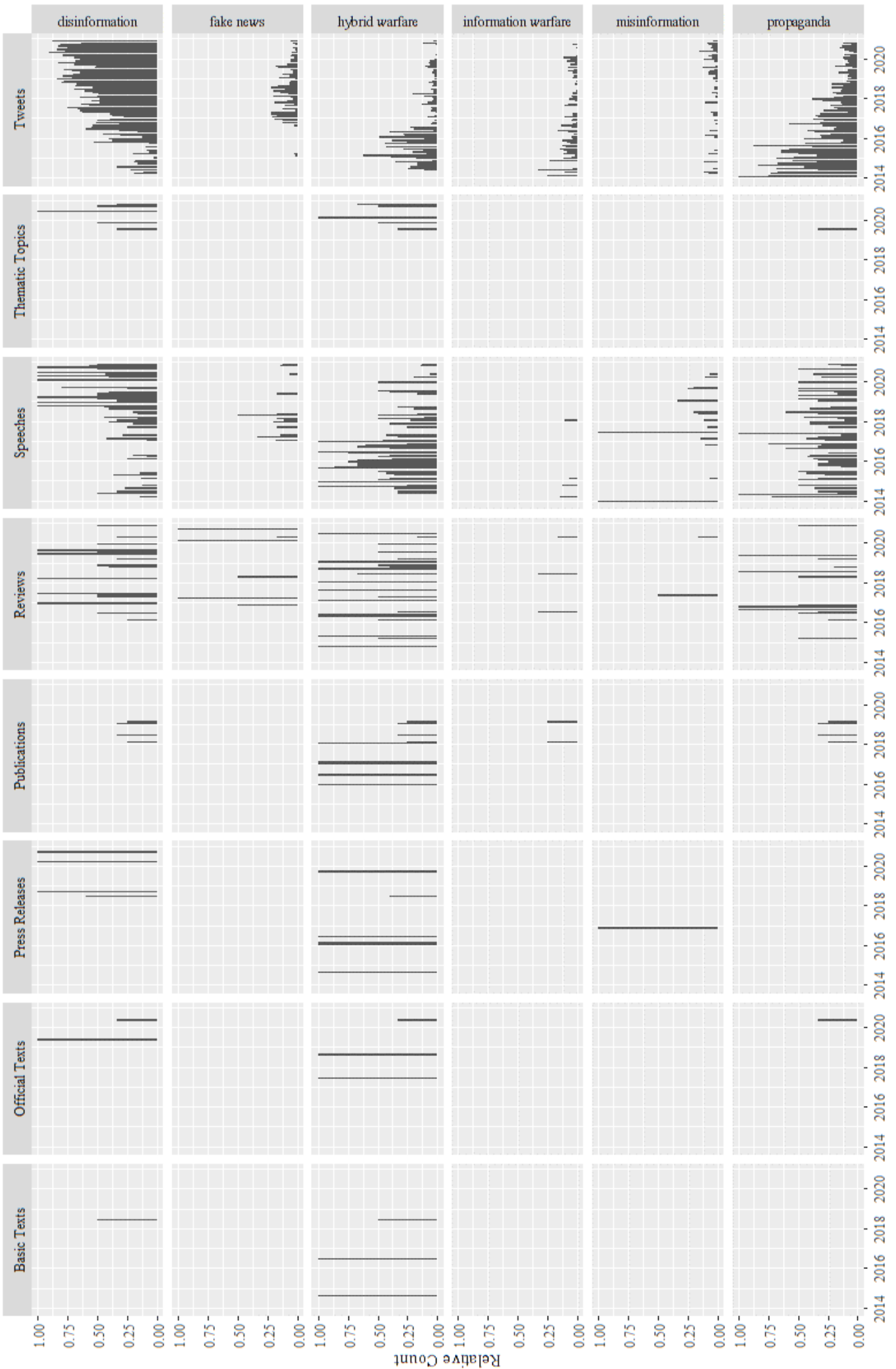

Figure 2: Longitudinal temporal histogram of top keywords (disinformation, fake news, hybrid warfare, information warfare, misinformation, propaganda) as they appear in NATO texts 

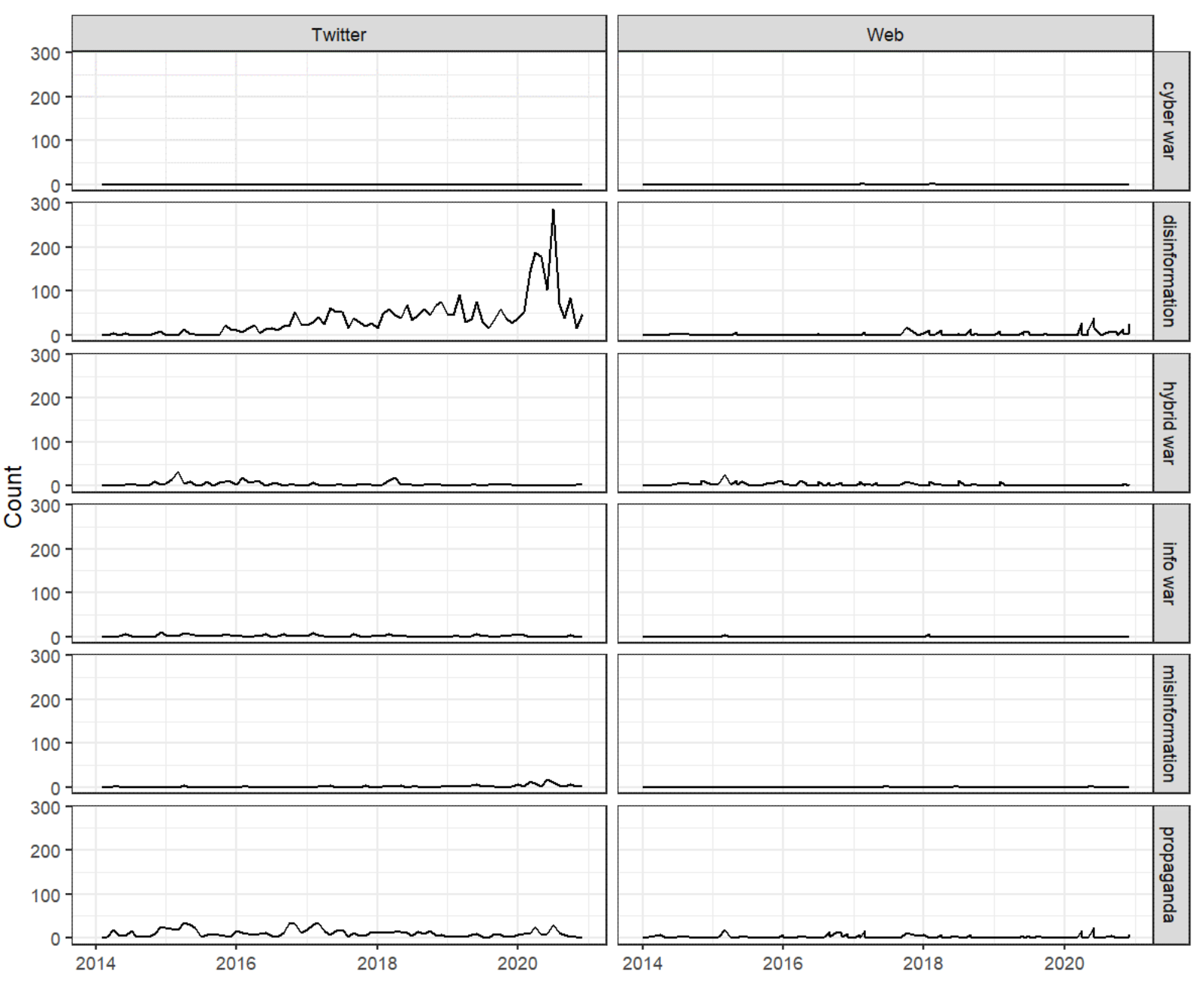

Figure 3: Longitudinal temporal frequency of top keywords as they appear in NATO Twitter texts vs official documents

representations use this word the most, Latvia (@LV_NATO), Lithuania (@LitdelNATO), United States (@USNATO), Ukraine (@NATOinUkraine) and Germany (@GermanyNATO) stand out the most.

Our 'keyness measures' (Marchi, Taylor, 2018) (two-by-two frequencies of words within a sample) indicate that while NATO's official documents are more general with regard to its strategic word choices, NATO's tweets are overwhelmingly focused on 'disinformation', 'propaganda' and 'fake news' terms within the context of Russia ('pro-Kremlin', 'Russian' and 'Kremlin' designations).

\section{Unsupervised Structural Topic Model Results}

For the structural topic models, we used the stm package for R, developed by Molly Roberts, Brandon Stewart and Dustin Tingley (Roberts et al., 2019). Stm was developed as part of its developers quest to come up with a methodological tool that would allow them to generate causal inferences from text data. By measuring document-level covariate measures, it introduces a new form of qualitative inference and within- 


\begin{tabular}{lll}
\hline Screen Name & $\mathrm{N}$ & Ratio \\
\hline STRATCOMCOE & 500 & 19.01 \\
NATOmoscow & 417 & 15.86 \\
NATOBrazeB & 398 & 15.13 \\
NATOpress & 231 & 8.78 \\
LV_NATO & 119 & 4.52 \\
LitdelNATO & 104 & 3.95 \\
NATORomeroC & 90 & 3.42 \\
NATO & 89 & 3.38 \\
USNATO & 82 & 3.12 \\
NATOinUkraine & 74 & 2.81 \\
GermanyNATO & 62 & 2.36 \\
CanadaNATO & 52 & 1.98 \\
ItalyatNATO & 45 & 1.71 \\
PLinNATO & 43 & 1.63 \\
UKNATO & 42 & 1.60 \\
SwedenNato & 35 & 1.33 \\
Slovakia_NATO & 31 & 1.18 \\
NATODepSpox & 27 & 1.03 \\
SHAPE_NATO & 23 & 0.87 \\
SpainNATO & 23 & 0.87 \\
\hline
\end{tabular}

Table 3: Official NATO-affiliated accounts sorted by the ratio of disinformation-related tweets as part of their aggregate tweets

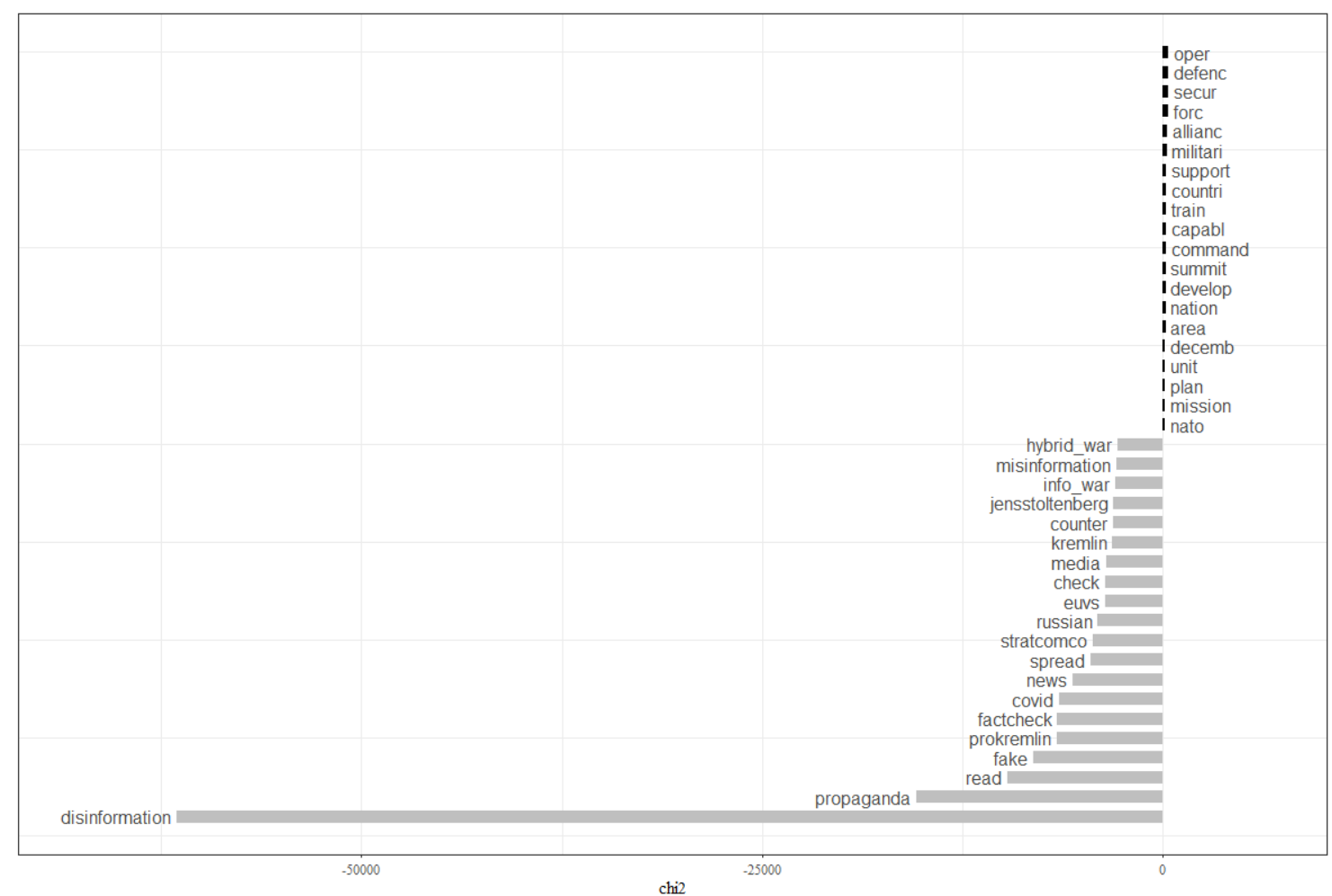

Figure 4: Keyness (textual context - most and least likely correlations) graph of tweets and official documents relative to keyword 'disinformation'. Highest likelihood keyword is itself: 'disinformation'. After that, the plot shows ranked keywords from bottom to up, according to how frequently they appear with the main keyword 'disinformation'. 


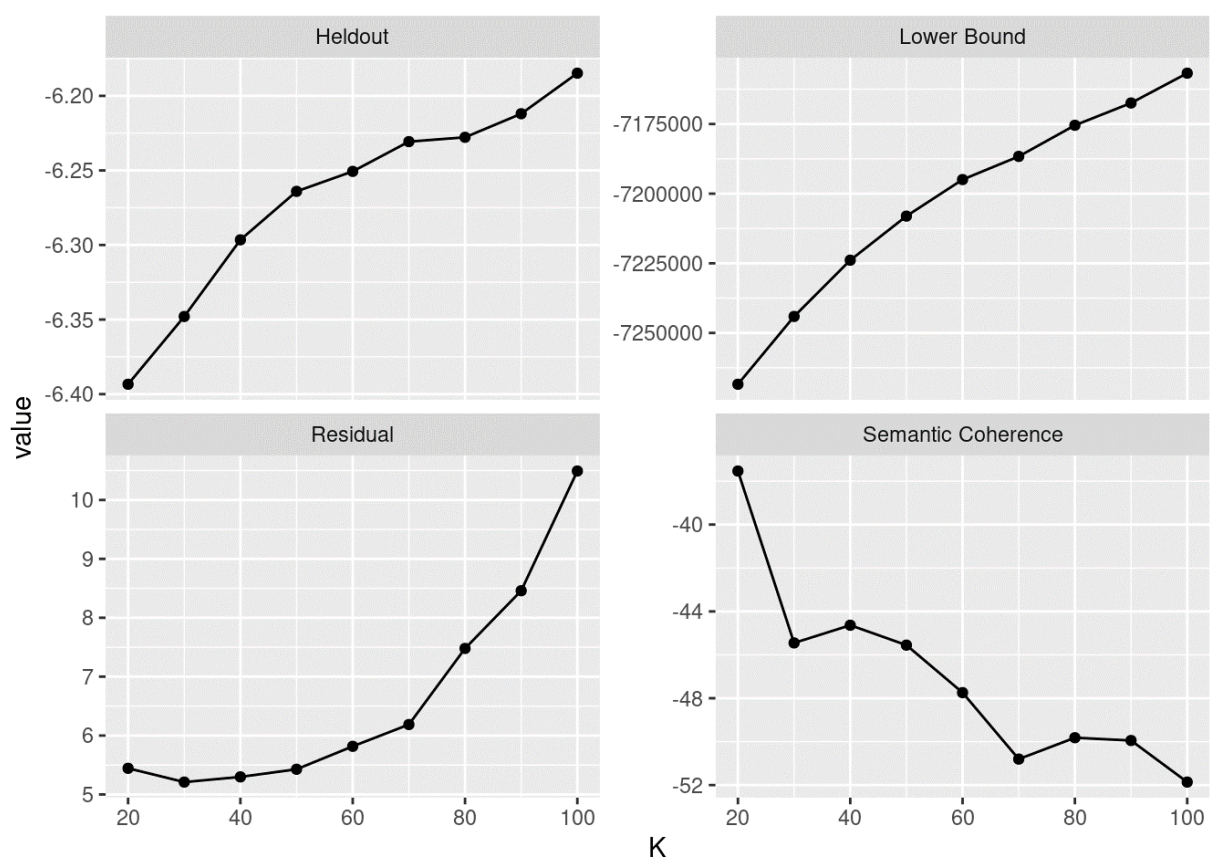

Figure 5: Topic count graph demonstrating the optimization rationale for our stm algorithm's choice of 50 topic models. The 'K-value' shows the optimum number of 'structural topic models' the algorithm has to go through the text to find the optimum semantic coherence. In other words, the K number designates the optimum number of structural topic models in texts that have the highest statistical coherence coefficients. Often, $\mathrm{K}$ values are assigned by the programmer and an optimum number gets eyeballed after several trial and error runs. K-value optimization uses machine learning to iterate through the text multiple times to find the optimum $\mathrm{K}$-value by statistical clustering of frequently collocated word combinations.

text estimation algorithms for better topic correlations. This ultimately helps us generate more accurate topic associations and themes within complex, lengthy documents.

Our unsupervised machine learning tests within NATO documents and tweets containing disinformationrelated trigger words yielded 50 topic models with an optimum combination of semantic coherence and heldout values. Out of these 50 models, our algorithm found that 10 of them had greater higher semantic salience and thus, had a statistically higher likelihood of forming a coherent 'topic'. Since not all word combination likelihoods imply a theme, K-means clustering is required to measure co-occurrence likelihood of words that make of a topic, in relation to the statistical significance of other topics (Roberts et al., 2014; Wang et al., 2011). These are the topics classified and numbered by our stm algorithm as 1, 4, 7, 9, 14, 16, $23,35,39,47$.

Topic 1 demonstrates the over-reliance on the term 'disinformation' as the dominant discursive anchor for NATO documents, mostly correlating with keywords associated with its spread, the role of fact-checking and misinformation, which is a less-used term. Topic 16 demonstrates that the term 'Russian' is highly correlated with 'fake' and 'news' terms, within the context of info(rmation)_war(fare), as well as 'troll'. The second most salient model is Topic 23, which builds around the keyword 'propaganda'. This model correlates most significantly with the n-gram clusterings: 'strateg_', 'fact', 'truth' and 'counter', suggesting that such emphasis is generally made within the context of combating external efforts of propaganda. Third 


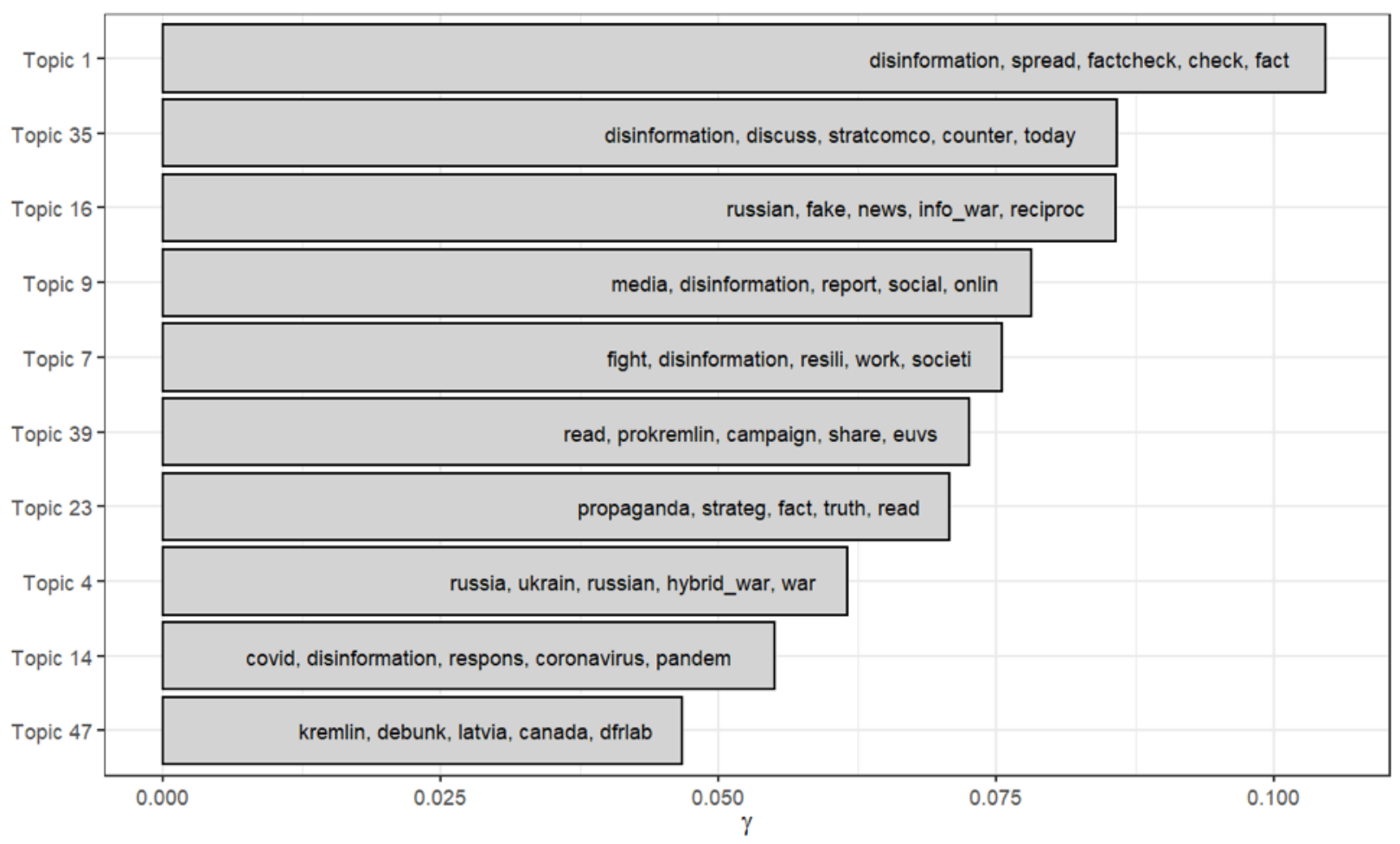

Figure 6: Top ten topics by prevalence and gamma-values ( ) that measure their levels of contribution to each topic

most salient Topic Model is 4, built around Russia, and the n-grams 'Ukrain_', 'hybrid_war', 'Putin', and 'Moscow'. At least within the context of Russian military involvement in Crimea and Donbass, NATO has largely relied on the term 'hybrid warfare', instead of 'disinformation', or 'misinformation', suggesting that it doesn't consider this military entanglement within the context of 'disinformation'.

To understand NATO's most active institution that deals with disinformation defense, Topic 35 is instructive. There, the keyword 'disinformation' correlates with STRATCOMCOE (NATO Strategic Communications Centre of Excellence in Riga, Latvia), and RigaStratCom revealing NATO's frontier defense mechanism of choice in issues related to disinformation. This is in line with Topic 30, where geographies correlated with our 'disinformation' keyword cluster reveals 'europ__, 'baltic', 'german_, 'danger' and 'prepar_.', hinting at NATO's perceived geographic vulnerability against disinformation attempts. A secondary vulnerability cluster emerges in Topic 49, where 'lithuania', 'estonia', 'japan', 'poland', and 'baltic' designations correlate with 'target' and 'defens_.' n-grams (Damashek, 1995) 1. In Topic 14, we discover the emergence of COVID-related disinformation issues, although the correlated terms are not yet sufficient to infer a political trend of preference.

Longitudinal topic frequency analysis of Topic 1 shows that the keyword 'disinformation' indeed enters into the NATO lexicon after the Russian military involvement in Ukraine. However, we observe a clear

\footnotetext{
${ }^{1}$ In computational linguistics, an n-gram is a continous sequence of 'n-items' (letters, words) that form a part of speech or text. Often, n-grams are used for 'stemming', reducing words to their simplest base. For example, words 'attacked', 'attacking', 'attacker' derive from the n-gram stem 'attac_.'
} 

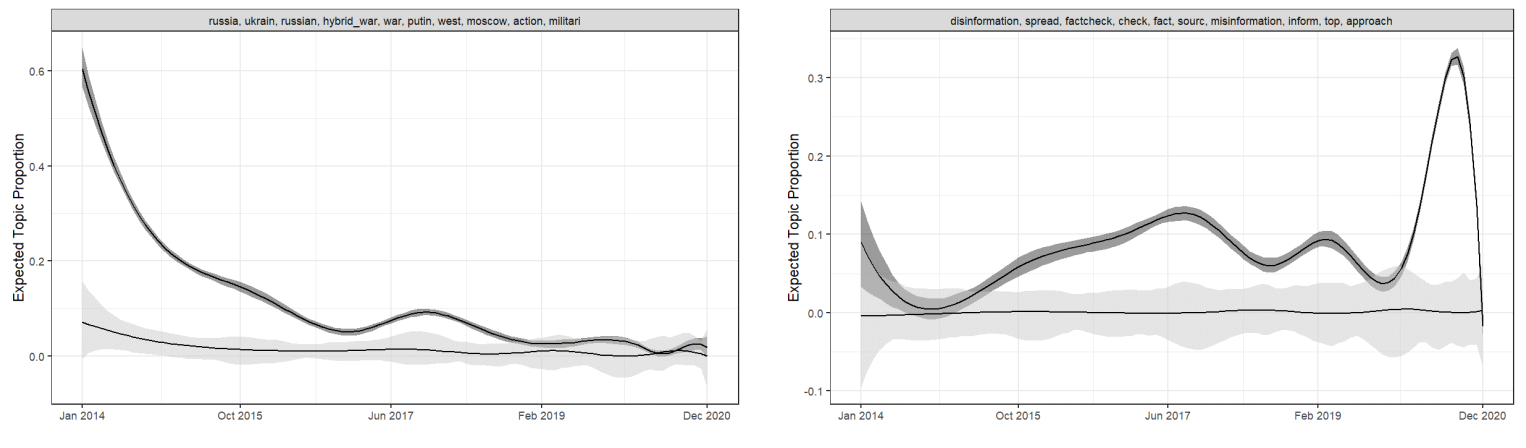

Tweet Web
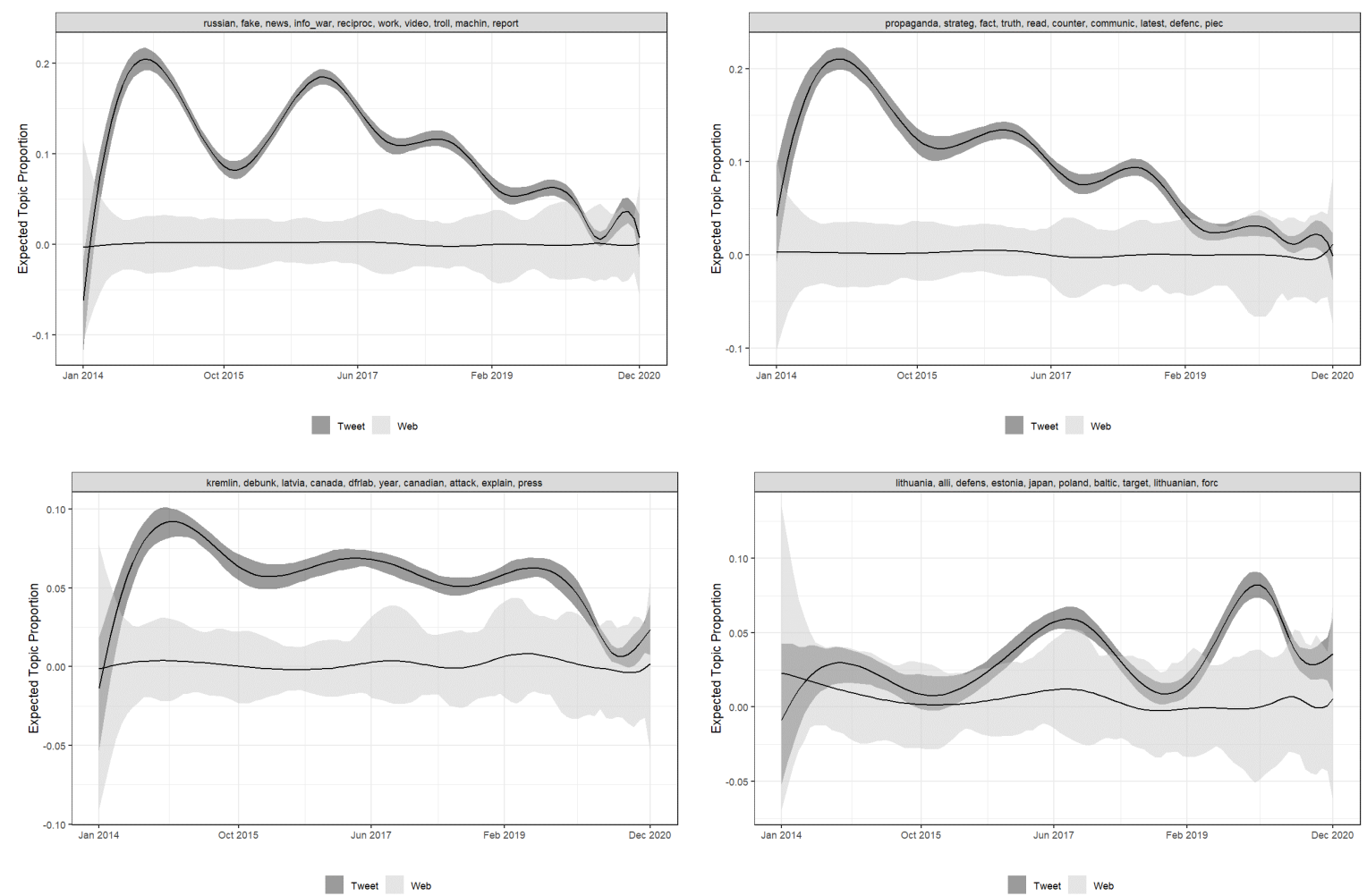

Figure 7: Top ten topics by prevalence and gamma-values ( ) that measure their levels of contribution to each topic

difference between NATO official texts (web) that don't prefer this term, versus NATO tweets that overwhelmingly rely on it. The peak in early 2014 is followed by a second peak after late-2016, possibly owing to the US elections, reaching its all-time peak in 2020, predictably due to COVID-related concerns. Topic 4 demonstrates how Russian involvement in Ukraine, as well as the 'hybrid war' narrative becomes less popular over time. Despite its significant salience in NATO tweets and a slight reference in its documents in 2014, these references largely disappear from NATO's discursive attention zone by 2016.

Tendency to pinpoint Russia in statements containing 'fake news', 'troll' and 'information warfare' keywords is once again clearest in tweets compared to official statements, as seen in Topic 16. This tendency peaks once during Russian military operation in Ukraine, for a second time around the US elections in 2016 , 
gradually disappearing from NATO's Twitter focus gradually after 2017 with a brief third peak around the poisoning of Sergei and Yulia Skripal in Salisbury, UK in March 2018. Same goes for Topic 23, which focuses on terms correlated with 'propaganda'. It peaks with the Russian involvement in Ukraine, marking a second brief peak around the US elections in 2016 and a third peak around the Skripal poisoning incident in 2018, later disappearing from NATO lexicon. NATO's reference to the Baltics and Germany as potential vulnerabilities against disinformation and hybrid war, Topic 30 produces a more varied picture. Here, we observe a significant and comparable activity within NATO tweets and official documents alike. Both NATO tweets and official documents follow similar curves around the same periods (Ukraine war, US elections in 2016, Skripal poisoning and COVID onset in March 2020), suggesting that such geographic vulnerabilities aren't new, and carry on significant strategic baggage from the past. Indeed, as Topics 47 and 49 also demonstrates, concerns and vulnerabilities around Canada, Lithuania, Estonia, Poland and the Baltic states are emphasized continuously both in NATO official documents and in tweets.

To sum up, NATO's discourse on disinformation presents a discursive continuity and is broadly in line with its securitization preferences prior to the popularization of the terms 'fake news' or 'information operations'. By leveraging a buzzword that has mainstream popularity, NATO's discursive efforts refocus the alliance's strategic agenda back on Russia, and semantically clusters these securitization moves on existing competition areas with Moscow. Since securitization is the process by which regular events, actors and phenomena are elevated into a policy frame that requires special measures, NATO's disinformation discourse directly fits into the theoretical spectrum. NATO's 2018 Brussels Summit Declaration and the 2019 London Declaration both prioritized disinformation as a major, strategic-level alliance threat and combating information warfare have been integrated into NATO military exercises since 2017. NATO has been running wargames that focus on coordinated, Russian-origin disinformation campaigns against NATO battlegroups in Latvia, Lithuania, and Poland and has been investing in the establishment of new digital countermeasure labs (NATO, 2020). In other words, as a successful securitization effort, disinformation has been receiving ample attention, resources and cohesion-building initiatives within the NATO framework. As part of this strategy, NATO's securitization efforts have a clear securitizing agent (alliance), existential threat (Russian-origin information warfare), a referent object (alliance cohesion, electoral integrity) and an audience (international public opinion), along with new doctrinal changes and investment schemees.

\section{Discussion and Conclusion}

Our analysis has shown that NATO has developed two disinformation-related communication strategies for two outlets: a more up-to-date and faster-developing threat discourse for its Twitter presence, and a more traditional, slow-moving threat presence visible in its official documents. This is particularly interesting and acutely visible in more 20th century military topics like command and control cohesion, missile defense, air defense architecture, naval defense, satellites and military intelligence-related topics that are more frequently mentioned in official documents, and much less referenced on Twitter. However, the overwhelming majority of disinformation, misinformation, hybrid war, information warfare and fake news-related communication 
topics are securitized on Twitter. This shows the emergence of two NATO discourses: one for its official documents, and one for its social media presence and messaging.

The advent of digital communication technologies and social media has been significant for the evolution of securitization. Since securitization entails production and dissemination of insecurity frames through discursive networks, a more dynamic, interconnected information ecosystem is more conducive for collective meaning-making. On social media, the formation of insecurity processes are more rapid and interactive, and are able to influence and alter traditional, boring securitizing acts of elites. To that end, media outlets like Twitter provide a more interactive and fast-paced securitizing environment where elites and non-elites can set the security agenda and mobilize masses. The most clear expression of this novel medium, as demonstrated in our results, is that NATO's Twitter securitization efforts change much faster, and spread more widely than traditional outlets like official speeches, texts and reports.

This could be interpreted in two ways: first, that NATO may prefer securitizing disinformation exclusively on Twitter, since such threats are generally more visible and debated on social media platforms. The second interpretation is that NATO's official statements and documents could largely be focusing on macrolevel doctrinal issues that pose a direct military security threat to its members, rather than disinformation, which is a nuisance, but poses no direct military threat. Since disinformation is being discussed in contemporary policy debates within the context of electoral integrity and social polarization, their actual military relevance may be less relevant to be taken into account in formal NATO documents. In either case, our study of the NATO lexicon demonstrated that disinformation and related terms are constructed as uniquely 'Russian' nuisances. This isn't surprising since most of these terms - at least their digital interpretations - have entered the NATO lexicon after the Russian military involvement in eastern Ukraine and Crimea. However, since then, Russia remained the only country against which NATO has constructed its disinformation narratives, indicating that Russia is NATO's sole disinformation concern. Although very recently China has emerged as a runner-up country within the context of COVID-related disinformation concerns, Russia is largely the main threat in NATO's lexicon. This could be counterproductive to long-term NATO efforts to combat disinformation, given the global prevalence of fake news and information meddling. While Russian disinformation efforts are observably valid, cornering a universal problem like disinformation into the limited space of NATO's interactions with a single country may lead to conceptual contraction. This in turn, may prevent NATO from mobilizing full alliance resources against disinformation, defined as a global and universal problem.

Overall, our analysis has shown that NATO still defines its security identity against Russia, and there isn't a significant shift in NATO's securitization dynamics since the Cold War, evidenced by our comparative analysis of older and newer NATO texts. Although Chinese disinformation attempts have also begun to enter into NATO threat language, NATO's primary discursive security identity continues to develop against and around Russia. This is most evident in our longitudinal analysis or pre- and post-2014 documents that prioritize Russia as a threat alike, implying that it is not really disinformation or fake news agenda that is rendering Russia a threat for NATO. This suggests the hypothesis that even if technologies change, NATORussia rivalry will remain securitized the same way. In other words, contemporary disinformation and fake 
news agenda is a continuation of the same NATO-Russia rivalry - at least in discursive form - through newer mediums.

\section{References}

Andrejevic Mark. Infoglut: How Too Much Information Is Changing the Way We Think and Know. 2013.

Balzacq Thierry, Léonard Sarah, Ruzicka Jan. 'Securitization' Revisited: Theory and Cases // International Relations. XII 2016. 30, 4. 494-531. Publisher: SAGE Publications Ltd.

Baum Matthew A., Potter Philip B. K. Media, Public Opinion, and Foreign Policy in the Age of Social Media // The Journal of Politics. IV 2019. 81, 2. 747-756. Publisher: The University of Chicago Press.

Baumann Mario. 'Propaganda Fights' and 'Disinformation Campaigns': the Discourse on Information Warfare in Russia-West Relations // Contemporary Politics. V 2020. 26, 3. 288-307. Publisher: Routledge _eprint: https://doi.org/10.1080/13569775.2020.1728612.

Bennett W Lance, Livingston Steven. The Disinformation Order: Disruptive Communication and the Decline of Democratic Institutions // European Journal of Communication. IV 2018. 33, 2. 122-139. Publisher: SAGE Publications Ltd.

Bouvier Gwen, Machin David. Critical Discourse Analysis and the Challenges and Opportunities of Social Media // Review of Communication. VII 2018. 18, 3. 178-192. Publisher: Routledge_eprint: https://doi.org/10.1080/15358593.2018.1479881.

Bradshaw Samantha, Howard Philip N. The Global Organization of Social Media Disinformation Campaigns // Journal of International Affairs. 2018. 71, 1.5. 23-32.

Buzan Barry, Buzan Research Professor of International Studies Centre for the Study of Democracy Barry, Wver Ole, Wæver Ole, Wilde Jaap de. Security: A New Framework for Analysis. 1998. UK. Google-BooksID: 7UdXtAEACAAJ.

Buzan Barry, Wæver Ole. Macrosecuritisation and Security Constellations: Reconsidering Scale in Securitisation Theory // Review of International Studies. 2009. 35, 2. 253-276. Publisher: Cambridge University Press.

Cour Christina la. Theorising Digital Disinformation in International Relations // International Politics. VIII 2020. 57, 4. 704-723.

Damashek Marc. Gauging Similarity with n-Grams: Language-Independent Categorization of Text // Science. II 1995. 267, 5199. 843-848. Publisher: American Association for the Advancement of Science.

DiMaggio Paul. Adapting Computational Text Analysis to Social Science (and vice versa) // Big Data \& Society. XII 2015. 2, 2. 2053951715602908. Publisher: SAGE Publications Ltd.

Farkas Johan, Schou Jannick. Fake News as a Floating Signifier: Hegemony, Antagonism and the Politics of Falsehood // Javnost - The Public. VII 2018. 25, 3. 298-314. Publisher: Routledge _eprint: https://doi.org/10.1080/13183222.2018.1463047. 
Freelon Deen, Wells Chris. Disinformation as Political Communication // Political Communication. III 2020. 37, 2. 145-156. Publisher: Routledge_eprint: https://doi.org/10.1080/10584609.2020.1723755.

Galeotti Mark. The Mythical 'Gerasimov Doctrine' and the Language of Threat // Critical Studies on Security. V 2019. 7, 2. 157-161. Publisher: Routledge _eprint: https://doi.org/10.1080/21624887.2018.1441623.

Giles Jim. Computational Social Science: Making the Links // Nature. VIII 2012. 488, 7412. 448-450. Number: 7412 Publisher: Nature Publishing Group.

Grinberg Nir, Joseph Kenneth, Friedland Lisa, Swire-Thompson Briony, Lazer David. Fake news on Twitter during the 2016 U.S. Presidential Election // Science (New York, N.Y.). I 2019. 363, 6425. 374-378.

Guess Andrew M., Lyons Benjamin A. Misinformation, Disinformation, and Online Propaganda // Social Media and Democracy: The State of the Field, Prospects for Reform. Cambridge: Cambridge University Press, 2020. 10-33. (SSRC Anxieties of Democracy).

Hong Liangjie, Davison Brian D. Empirical study of topic modeling in Twitter // Proceedings of the First Workshop on Social Media Analytics - SOMA '10. Washington D.C., District of Columbia: ACM Press, 2010. 80-88.

Jack Caroline. Lexicon of Lies: Terms for Problematic Information // Data \& Society. 2017. 3, 22. 1094-1096. Publisher: Data \& Society Research Institute.

Khaldarova Irina, Pantti Mervi. Fake News // Journalism Practice. X 2016. 10, 7. 891-901. Publisher: Routledge_eprint: https://doi.org/10.1080/17512786.2016.1163237.

Knudsen Olav F. Post-Copenhagen Security Studies: Desecuritizing Securitization // Security Dialogue. IX 2001. 32, 3. 355-368. Publisher: SAGE Publications Ltd.

Krippendorff Klaus. Measuring the Reliability of Qualitative Text Analysis Data // Quality \& Quantity. XII 2004. 38, 6. 787-800.

Kurowska Xymena, Reshetnikov Anatoly. Neutrollization: Industrialized trolling as a pro-Kremlin strategy of desecuritization // Security Dialogue. X 2018. 49, 5. 345-363. Publisher: SAGE Publications Ltd.

Lanoszka Alexander. Disinformation in International Politics // European Journal of International Security. VI 2019. 4, 2. 227-248. Publisher: Cambridge University Press.

Liang Hai, Fu King-wa. Testing Propositions Derived from Twitter Studies: Generalization and Replication in Computational Social Science // PLOS ONE. VIII 2015. 10, 8. e0134270. Publisher: Public Library of Science.

Lipizzi Carlo, Dessavre Dante, Iandoli Luca, Marquez Jose. Towards Computational Discourse Analysis: A Methodology for Mining Twitter Backchanneling Conversations // Computers in Human Behavior. XI 2016. 64. 782-792.

Lysenko Volodymyr, Brooks Catherine. Russian information troops, disinformation, and democracy // First Monday. IV 2018. 23, 5. 
Mälksoo Maria. Countering Hybrid Warfare as Ontological Security Management: the Emerging Practices of the EU and NATO // European Security. VII 2018. 27, 3. 374-392. Publisher: Routledge_eprint: https://doi.org/10.1080/09662839.2018.1497984.

Marchi Anna, Taylor Charlote. Corpus Approaches to Discourse: A Critical Review. New York: Routledge, 2018. 1st.

Mas-Manchón Lluís, Guerrero-Solé Frederic, Ramon Xavier, Grande Laura. Patriotic Journalism in Fake News Warfare: El País' Coverage of the Catalan Process // The Political Economy of Communication. III 2021. 8, 2. Number: 2 .

Maweu Jacinta Mwende. "Fake Elections"? Cyber Propaganda, Disinformation and the 2017 General Elections in Kenya // African Journalism Studies. X 2019. 40, 4. 62-76. Publisher: Routledge _eprint: https://doi.org/10.1080/23743670.2020.1719858.

Mejias Ulises A, Vokuev Nikolai E. Disinformation and the media: the case of Russia and Ukraine // Media, Culture \& Society. X 2017. 39, 7. 1027-1042. Publisher: SAGE Publications Ltd.

Monsees Linda. 'A war against truth' - understanding the fake news controversy // Critical Studies on Security. V 2020. 8, 2. 116-129. Publisher: Routledge_eprint: https://doi.org/10.1080/21624887.2020.1763708.

Morgan Susan. Fake News, Disinformation, Manipulation and Online Tactics to Undermine Democracy // Journal of Cyber Policy. I 2018. 3, 1. 39-43. Publisher: Routledge _eprint: https://doi.org/10.1080/23738871.2018.1462395.

NATO . NATO's approach to countering disinformation: a focus on COVID-19. 2020.

Neo Ric. When Would a State Crack Down on Fake News? Explaining Variation in the Governance of Fake News in Asia-Pacific // Political Studies Review. V 2021. 14789299211013984. Publisher: SAGE Publications.

Polletta Francesca, Callahan Jessica. Deep Stories, Nostalgia Narratives, and Fake News: Storytelling in the Trump Era // Politics of Meaning/Meaning of Politics: Cultural Sociology of the 2016 U.S. Presidential Election. Cham: Springer International Publishing, 2019. 55-73.

Renz Bettina. Russian Military Capabilities after 20 Years of Reform // Survival. V 2014. 56, 3. 61-84. Publisher: Routledge_eprint: https://doi.org/10.1080/00396338.2014.920145.

Roberts Margaret E, Stewart Brandon M, Tingley Dustin. stm: R Package for Structural Topic Models // Journal of Statistical Software. 2019. 91, 1. 1-40.

Roberts Margaret E., Stewart Brandon M., Tingley Dustin, Lucas Christopher, Leder-Luis Jetson, Gadarian Shana Kushner, Albertson Bethany, Rand David G. Structural Topic Models for Open-Ended Survey Responses // American Journal of Political Science. X 2014. 58, 4. 1064-1082.

Roberts Margaret E, Tingley Dustin, Stewart Brandon M, Airoldi Edoardo M. The Structural Topic Model and Applied Social Science // ICONIP2013. South Korea: Daegu, 2013. 4.

Ross Andrew, Rivers Damian J. Discursive Deflection: Accusation of "Fake News" and the Spread of Misand Dis-information in the Tweets of President Trump // Social Media + Society. IV 2018. 4, 2. 1-12. 
Saurwein Florian, Spencer-Smith Charlotte. Combating Disinformation on Social Media: Multilevel Governance and Distributed Accountability in Europe // Digital Journalism. VII 2020. 8, 6. 820-841. Publisher: Routledge _eprint: https://doi.org/10.1080/21670811.2020.1765401.

Sinovets Polna, Renz Bettina. Russia's 2014 Military Doctrine and beyond: Threat Perceptions, Capabilities and Ambitions // NATO Research Papers. VII 2015.

Smith Christopher A. Weaponized Iconoclasm in Internet Memes Featuring the Expression 'Fake News' // Discourse \& Communication. VI 2019. 13, 3. 303-319. Publisher: SAGE Publications.

Stritzel Holger, Chang Sean C. Securitization and Counter-Securitization in Afghanistan // Security Dialogue. XII 2015. 46, 6. 548-567. Publisher: SAGE Publications Ltd.

Tan Netina. Electoral Management of Digital Campaigns and Disinformation in East and Southeast Asia // Election Law Journal: Rules, Politics, and Policy. VI 2020. 19, 2. 214-239. Publisher: Mary Ann Liebert, Inc., publishers.

Tandoc Edson C., Lim Zheng Wei, Ling Richard. Defining "Fake News" // Digital Journalism. II 2018. 6, 2. 137-153. Publisher: Routledge_eprint: https://doi.org/10.1080/21670811.2017.1360143.

Tong Chau, Gill Hyungjin, Li Jianing, Valenzuela Sebastián, Rojas Hernando. "Fake News Is Anything They Say!" - Conceptualization and Weaponization of Fake News among the American Public // Mass Communication and Society. IX 2020. 23, 5. 755-778. Publisher: Routledge _eprint: https://doi.org/10.1080/15205436.2020.1789661.

Tucker Joshua A., Guess Andrew, Barbera Pablo, Vaccari Cristian, Siegel Alexandra, Sanovich Sergey, Stukal Denis, Nyhan Brendan. Social Media, Political Polarization, and Political Disinformation: A Review of the Scientific Literature. Rochester, NY, III 2018.

Van Duyn Emily, Collier Jessica. Priming and Fake News: The Effects of Elite Discourse on Evaluations of News Media // Mass Communication and Society. I 2019. 22, 1. 29-48. Publisher: Routledge _eprint: https://doi.org/10.1080/15205436.2018.1511807.

Wallach Hanna M. Topic Modeling: Beyond Bag-of-Words // Proceedings of the 23rd international conference on Machine learning - ICML ’06. Pittsburgh, Pennsylvania: ACM Press, 2006. 977-984.

Wang Hongning, Zhang Duo, Zhai ChengXiang. Structural Topic Model for Latent Topical Structure Analysis // Proceedings of the 49th Annual Meeting of the Association for Computational Linguistics: Human Language Technologies. Portland, Oregon, USA: Association for Computational Linguistics, VI 2011. $1526-1535$.

Williams Michael C. Words, Images, Enemies: Securitization and International Politics // International Studies Quarterly. XII 2003. 47, 4. 511-531. 